\title{
Dynamic overlap concentration scale of active colloids
}

\author{
Stewart A. Mallory $\odot,{ }^{1, *}$ Ahmad K. Omar, ${ }^{2, \dagger}$ and John F. Brady ${ }^{3, *}$ \\ ${ }^{1}$ Department of Chemistry, The Pennsylvania State University, University Park, Pennyslvania 16802, USA \\ ${ }^{2}$ Department of Materials Science and Engineering, University of California, Berkeley, California 94720, USA \\ ${ }^{3}$ Division of Chemistry and Chemical Engineering, California Institute of Technology, Pasadena, California 91125, USA
}

(Received 13 September 2020; revised 22 August 2021; accepted 6 October 2021; published 26 October 2021)

\begin{abstract}
By introducing the notion of a dynamic overlap concentration scale, we identify additional universal features of the mechanical properties of active colloids. We codify these features by recognizing that the characteristic length scale of an active particle's trajectory, the run length, introduces a concentration scale $\phi^{*}$. Large-scale simulations of repulsive active Brownian particles (ABPs) confirm that this run-length dependent concentration, the trajectory-space analog of the overlap concentration in polymer solutions, delineates distinct concentration regimes in which interparticle collisions alter particle trajectories. Using $\phi^{*}$ and concentration scales associated with colloidal jamming, the mechanical equation of state for ABPs collapses onto a set of principal curves that contain several overlooked features. The inclusion of these features qualitatively alters previous predictions of the behavior for active colloids, as we demonstrate by computing the spinodal for a suspension of purely repulsive ABPs. Our findings suggest that dynamic overlap concentration scales should help unravel the behavior of active and driven systems.
\end{abstract}

DOI: 10.1103/PhysRevE.104.044612

\section{INTRODUCTION}

Pressure continues to be a topic of fundamental interest in active matter [1-15], an interest motivated, in part, by the tantalizing prospect that mechanical equations of state (EOS) will play as necessary a role in active matter as they do in equilibrium theory. Pressure has already proven to be a salient metric for rationalizing the often-complex behavior of active suspensions ranging from instabilities exhibited by expanding bacterial droplets [16], vesicles filled with active particles [17-20], active depletion [21-23], and the dynamics of colloidal gels [24-26], membranes [27], and polymers [28-30] immersed in a bath of active colloids. The burgeoning research on the nature of pressure in active systems has not only contributed to progress in understanding the behavior of active systems but has also played a central role in assessing the validity of new theoretical concepts for nonequilibrium systems [31-41]. There is no better example than the intense focus on the development of nonequilibrium theories for the phase behavior of active particles [4,42-51] — a crucial ingredient for many is an EOS.

Pressure in active systems remains challenging to characterize analytically in all but the most straightforward cases. These challenges are due to a unique nonlocal contribution to the pressure referred to as the swim pressure $[1,3]$. This nontraditional source of stress is entirely rooted in trajectory space, and unraveling its concentration dependence necessitates the difficult task of understanding how many-body

\footnotetext{
*sam7808@psu.edu

†aomar@berkeley.edu

$\$$ jfbrady@ caltech.edu
}

correlations alter particle trajectories. The driving force for much of the novel behaviors observed in active systems, including the phenomena of motility-induced phase separation (MIPS) [52-56], is due to this inherent nonequilibrium contribution to the pressure.

The swim pressure emerges from the constant and timeirreversible motion generated by the nonconservative selfpropelling force of active particles. Within the context of the active Brownian particle (ABP) model-a popular minimal model for studying active colloids-particles self-propel at a constant speed $U_{0}$ and undergo Brownian rotational diffusion with a characteristic reorientation time $\tau_{R}$. At long times, an ideal $\mathrm{ABP}$ executes a random walk with a trajectory correlation length $\ell_{0}=U_{0} \tau_{R}$, which we call the intrinsic run length. A dilute system of ABPs with number density $n$ exerts a swim pressure directly proportional to this intrinsic run length, $\Pi^{s} \sim n \ell_{0} U_{0}$. For more concentrated systems, interparticle interactions reduce the trajectory correlation length, resulting in a density-dependent effective run length $\ell<\ell_{0}$ and a swim pressure of $\Pi^{s} \sim n \ell U_{0}$. In the limit of maximal packing, ABPs cannot execute their athermal random walk resulting in a vanishingly small effective run length (and swim pressure) that should be independent of $\ell_{0}$ and depend only on geometric packing constraints.

This simple physical picture (see Fig. 1) suggests the existence of a concentration scale that controls the crossover between the dynamically distinct dilute and jammed regimes of active suspensions. In this article, using large-scale computer simulations, we identify this run-length dependent concentration $\phi^{*}$, which exhibits several compelling analogies to the powerful concept of the overlap concentration of equilibrium polymer solutions [57-59]. Using $\phi^{*}$ and concentration scales associated with colloidal jamming, the 


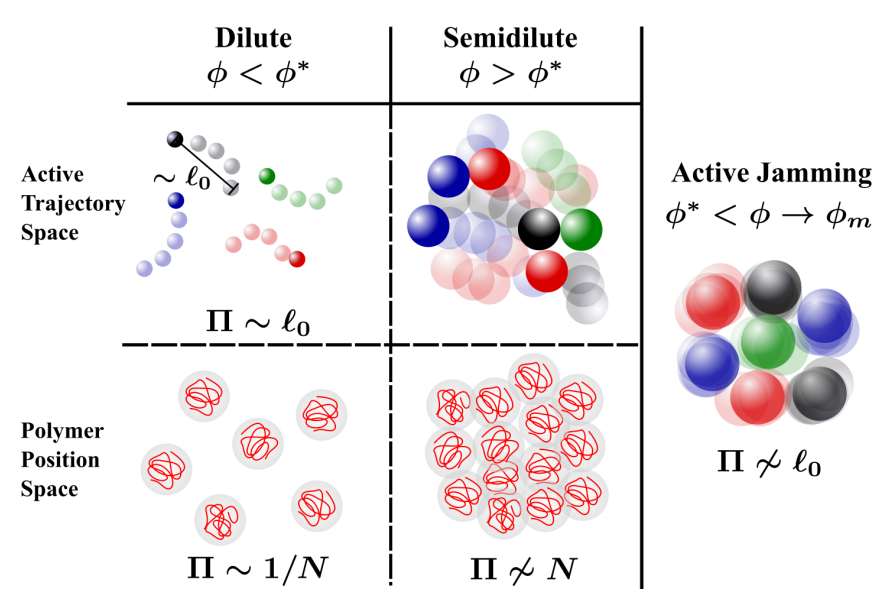

FIG. 1. Overlap concentration for polymer solutions and the proposed trajectory-space analog for active colloids. The scaling of the equilibrium osmotic pressure of the polymer solution with molecular weight $N$ and the pressure of active colloids with $\ell_{0}$ for different concentration regimes.

mechanical equation of state for ABPs collapses onto a single curve that contains overlooked features, the inclusion of which qualitatively alters other predictions of the phase behavior of ABPs. Moreover, the use of a dynamic overlap concentration scale may prove to be of great utility in constructing accurate nonequilibrium equations of state.

To unpack the concentration dependence of the pressure, we focus on one of the most widely studied active systems: purely repulsive active Brownian disks. By introducing a small degree of polydispersity in disk size, we ensure the system remains disordered for all concentrations. This behavior is in contrast to monodisperse active disks, which exhibit an order-disorder phase transition with a complex dependence on activity [60-62]. The small degree of polydispersity allows us to isolate the disordered branch of the equations of state, which will serve as a helpful reference system for active fluids with more complicated interactions and allows for the quantitative study of the interplay between activity and concentration.

\section{MODEL}

We consider a two-dimensional system of overdamped disks at fixed number density $n$ where each particle experiences a drag force $-\zeta \boldsymbol{U}$, a conservative interparticle force $\boldsymbol{F}^{\boldsymbol{c}}$, and an active self-propelling force $\boldsymbol{F}^{\boldsymbol{a}}=\zeta U_{0} \boldsymbol{q}$. The orientation of each particle $\boldsymbol{q}$ evolves independently and undergoes diffusive rotational dynamics with a characteristic reorientation time $\tau_{R}$. The sum of the forces results in a simple equation of motion for the particle velocity:

$$
\boldsymbol{U}=\left(\boldsymbol{F}^{a}+\boldsymbol{F}^{c}\right) / \zeta .
$$

The interparticle force $\boldsymbol{F}^{\boldsymbol{c}}$ arises from a Weeks-ChandlerAnderson (WCA) potential [63] characterized by a potential depth $\varepsilon$ and an average Lennard-Jones diameter $\sigma$. Particle diameters are drawn from a normal distribution with a standard deviation of $0.1 \sigma$. Importantly, as our active force is of finite amplitude, a sufficiently strong choice for the repulsive force $\boldsymbol{F}^{\boldsymbol{c}}$ will mimic a true hard-particle potential. A choice of $\varepsilon /\left(\zeta U_{0} \sigma\right)=100$ results in hard-disk statistics with an effective average particle diameter of $2^{1 / 6} \sigma$. In this hard-disk limit, the state of our system is independent of the amplitude of the active force and is fully described by two geometric parameters: the area fraction $\phi=n \pi\left(2^{1 / 6} \sigma\right)^{2} / 4$ and the dimensionless intrinsic run length $\ell_{0} / \sigma$. Using the GPUenabled HOOMD-BLUE software package [64], all simulations were conducted with 40000 particles and run for a minimum duration of $5000 \sigma / U_{0}$.

The total mechanical pressure $\Pi$ has two contributions: the collisional pressure $\Pi^{c}$ arising from conservative interparticle interactions and the swim pressure $\Pi^{s}$ generated by the active force. The collisional pressure follows from the standard micromechanical virial for conservative interactions $\Pi^{c}=n\left\langle\boldsymbol{x} \cdot \boldsymbol{F}^{c}\right\rangle / 2$ where $\boldsymbol{x}$ is the particle position and $\langle\cdots\rangle$ denotes an average over all particles. At steady state, $\Pi^{s}$ can be written in the impulse form $[12,13,47]$

$$
\Pi^{s}=n \frac{\zeta U_{0} \tau_{R}}{2}\langle\boldsymbol{q} \cdot \boldsymbol{U}\rangle .
$$

For an isotropic system of active particles free of aligning interaction, Eq. (2) is the pressure that the suspension would exert on a flat torque-free macroscopic boundary. Under these conditions, the total pressure exerted on the surroundings is simply the sum of the collisional and swim pressure $\Pi=$ $\Pi^{c}+\Pi^{s}$.

Equation (2) allows us to probe via simulation the effective run length of the particles directly, $\ell \equiv \tau_{R}\langle\boldsymbol{q} \cdot \boldsymbol{U}\rangle$, which is the actual correlation length of a particle's trajectory and a measure of the correlation between the orientation of a particle and the forces (both active and interparticle) acting upon it. Analytical expressions for the effective run length $\ell$ require solving a many-body dynamics problem in both position and orientation space [65-67] and have been derived in only a few asymptotic limits. Additionally, the nature of $\ell$ is further obfuscated as direct measurement is limited to a narrow region of $\left(\ell_{0}, \phi\right)$-state space where motility-induced phase separation is absent (i.e., outside of the MIPS phase envelope). Paradoxically, both the spinodal and binodal prediction requires knowledge of $\ell$ in regions where it cannot be directly measured. Extrapolation of $\ell$ into these mechanically forbidden regions requires a complete understanding of the trends in $\ell$ as the system approaches the critical $\ell_{0}$ for MIPS $\left(\ell_{0} / \sigma \approx 13\right)$. Figure $2(\mathrm{a})$ presents the complete $\phi$ dependence of $\ell$ in regions of $\left(\ell_{0}, \phi\right)$-state space where the system is homogeneous.

\section{DISCUSSION}

Our approach for isolating these trends is motivated by two observations. First, $\ell$ becomes increasingly independent of the intrinsic run length $\ell_{0}$ with increasing concentration. In the dilute limit, $\ell=\ell_{0}=U_{0} \tau_{R}$, while in the limit of maximal packing $\ell \approx 0$. Second, as $\ell_{0}$ increases, deviations from the dilute scaling prediction of $\ell$ occur at lower concentrations. Microrheology calculations for hard disks show the first-order correction to the effective run length is $\ell \approx \ell_{0}(1-\phi)$ in the limit of large $\ell_{0}[3,68]$. As shown in Fig. 2(b), this expression is in excellent agreement with our data for persistent ABPs 

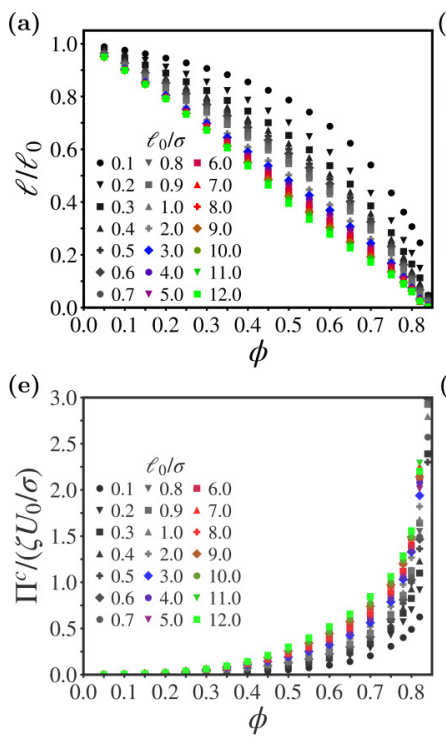
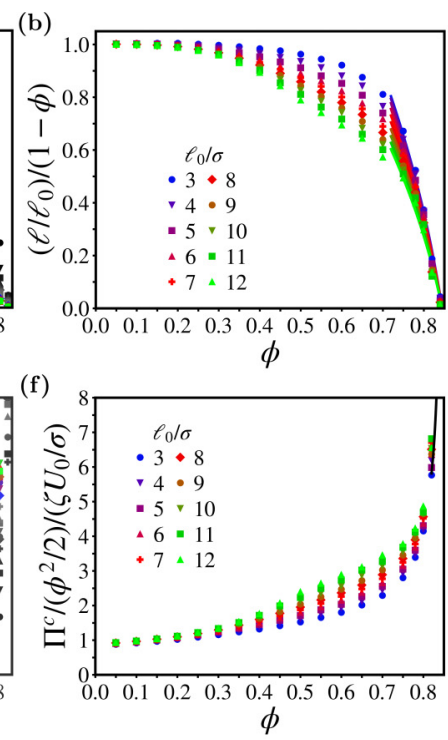
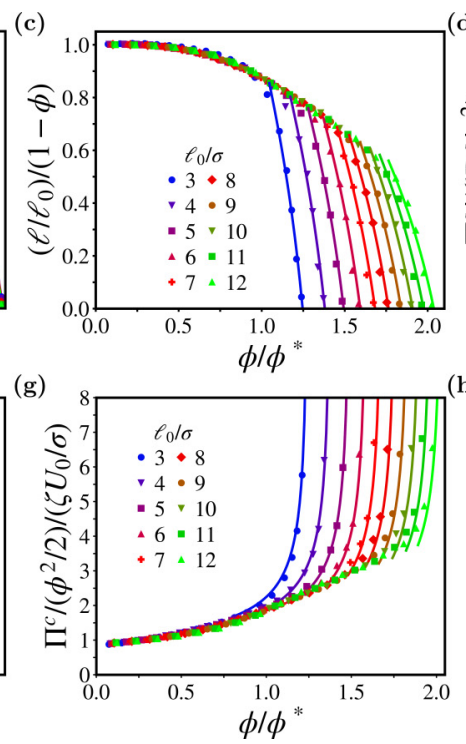

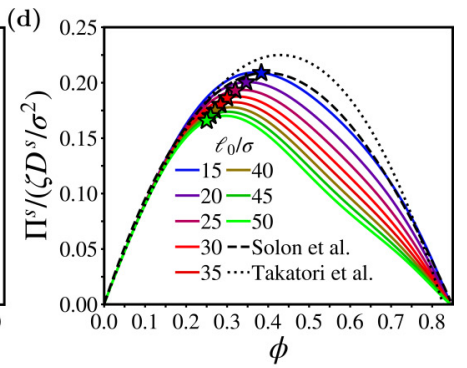

(h)

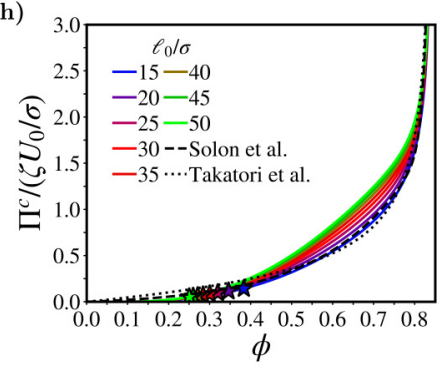

FIG. 2. Concentration dependence of the normalized effective run length (a) and $\Pi^{c}$ (e). The first-order corrections to the normalized run length (b) and $\Pi^{c}$ (f) in the dilute limit and the asymptotic scaling near maximal packing. Collapse of the dilute and semidilute regions for the effective run length (c) and $\Pi^{c}(\mathrm{~g})$ using the dynamic overlap concentration $\phi^{*}$. Lines represent our theoretical equations of state in the active jamming region. Scaling-informed predicted (d) swim (normalized by the ideal swim energy scale $\left.\zeta D^{s}=\zeta U_{0} \ell_{0} / 2\right)$ and (h) $\Pi^{c} \mathrm{~s}$ in the region of instability. The previously assumed forms of the swim pressure used by Takatori and Brady [42] and Solon et al. [47] are shown for comparison. For each activity curve, the identically colored star represents the location of $\phi^{*}$.

$\left(\ell_{0}>3 \sigma\right)$, and note the previously mentioned trend in the deviation from this dilute scaling as $\ell_{0}$ increases.

The dependence of the swim pressure on a single-particle length scale at low concentrations - the intrinsic run length $\ell_{0}$-and its independence from that length scale at high concentrations suggests a connection to equilibrium polymer solutions. There exist distinct concentration regimes for polymer solutions, corresponding to isolated (dilute) and overlapping chains (semidilute) [57-59]. The thermodynamic equations of state in each regime have different dependencies on single-chain properties. For example, the osmotic pressure of a dilute polymer solution is inversely proportional to the degree of polymerization $N$ (see Fig. 1). In the semidilute regime where chains strongly overlap, the individuality of each chain is lost, resulting in an osmotic pressure that is independent of the single-chain property $N$. The overlap concentration $\phi^{*}$ delineates the dilute and semidilute regimes and is the concentration at which polymer coils begin to overlap. The density of chains at $\phi^{*}$ is inversely proportional to the effective volume occupied by a single polymer chain $V^{\text {chain }}$.

A dynamical concentration scale for nonequilibrium systems is less straightforward to formulate than an equilibrium concentration scale. Nevertheless, as there is only a single intrinsic dynamical length scale for this system, $\ell_{0}$, it must play a crucial role in setting a concentration scale. The physical intuition here is that particles with larger intrinsic run lengths will collide more frequently than those with smaller run lengths at the same concentration. Our data reflect this scenario - the collisional pressure at a fixed concentration is an increasing function $\ell_{0}$ [see Fig. 2(e) and Supplemental Material [69] for pair correlation data]. It is this physical behavior that our overlap concentration can capture.

For ABPs, we seek the characteristic single-particle volume [area in two dimensions (2D)] $V^{\mathrm{ABP}}$ that plays the analogous role to the single-chain volume $V^{\text {chain }}$ for polymer solutions. In $2 \mathrm{D}$, a natural starting point is to consider the area swept out by an ideal $\mathrm{ABP}$ in a single reorientation time $\tau_{R}: V^{\mathrm{ABP}} \sim \ell_{0} \sigma$. For concentrations beyond $\phi^{*} \sim \sigma^{2} / V^{\mathrm{ABP}}$ particle trajectories overlap and collisions prevent ABPs from executing their intrinsic random walk of length $\ell_{0}$ (see Fig. 1). The single-particle volume given by $V^{\mathrm{ABP}} \sim \ell_{0} \sigma$ is likely an overestimate of the overlap probability. More realistically, we anticipate a weaker dependence on $\ell_{0}$ and introduce the more general definition $V^{\mathrm{ABP}} \sim \ell_{0}^{\lambda} \sigma^{2-\lambda}$ where $0<\lambda<1$ is a constant to be determined. The resulting overlap concentration $\phi^{*}$ can be written as

$$
\phi^{*} \sim\left(\frac{\sigma}{\ell_{0}}\right)^{\lambda} .
$$

If the overlap concentration were precisely analogous to equilibrium polymeric systems, one would find $\lambda=1$. In this scenario, when the areas swept out by the trajectories of two active particles in a time $\tau_{R}$ overlap, the particles are assumed to have collided. However, the spatial overlap of trajectories does not guarantee that the particles have collided, and we, therefore, expect $\lambda<1$. In effect, $\lambda$ provides a direct measure of the correlation between particle collisions and the intrinsic dynamic length scale $\ell_{0}$.

The dynamic overlap concentration $\phi^{*}$ proposed in Eq. (3) should delineate dilute and semidilute concentration regimes for all values of $\ell_{0}$. Using this criterion as a guide (see Supplemental Material [69]), we identify a significant dependence of the dynamic overlap concentration on the intrinsic run length with $\lambda \approx 0.353$ for our polydisperse system of active Brownian disks. In three dimensions (3D), it is less likely that trajectories overlap in comparison to $2 \mathrm{D}$, which should result in a smaller value of $\lambda$. We have conducted a systematic investigation of the EOS of active Brownian spheres, revealing 
that, consistent with our physical picture, $\lambda \approx 0.03$ (see Supplemental Material [69]).

For polydisperse ABPs, rescaling $\phi$ with $\phi^{*}$ allows for a collapse of $\ell$ in the dilute $\left(\phi / \phi^{*}<1\right)$ and semidilute $\left(\phi / \phi^{*} \geqslant\right.$ 1) regimes[see Fig. 2(c)]. Notably, for each $\ell_{0}$, deviations from the collapse begin to occur at a $\phi^{*}$-independent area fraction $\phi \approx 0.72$, indicative of an upper bound for our semidilute regime and the emergence of what we term as the active jamming regime.

As the system transitions to the active jamming regime, the run length tends to zero as the maximal packing $\phi_{m} \approx 0.845$ is approached. Furthermore, the run length becomes increasingly independent of $\ell_{0}$ as geometric packing constraints set the scale for particle motion. We find $\ell$ in the active jamming regime is well described by

$$
\ell / \ell_{0}=\mathcal{D}\left(1-e^{-\mathcal{E} / \ell_{0}}\right)\left(1-\phi / \phi_{m}\right),
$$

where $\mathcal{D}, \mathcal{E}>0$ are constant empirical fitting parameters [69]. As shown by the family of curves in Fig. 2(c), Eq. (4) accurately captures the deviation of $\ell$ from the semidilute regime as a function of $\ell_{0}$ while also capturing our physical asymptotic expectations. With increasing $\ell_{0}$, the effective run-length approaches a constant value set by the packing geometry, i.e., $\lim _{\ell_{0} \rightarrow \infty} \ell=\mathcal{D} \mathcal{E}\left(1-\phi / \phi_{m}\right)$. Previous EOS [42,47] which expressed $\ell=\ell_{0} f(\phi)$ (for large $\ell_{0}$ ) fail to capture this physical expectation and, in fact, find the unphysical result of $\lim _{\ell_{0} \rightarrow \infty} \ell=\infty$ for all $\phi$ unless $f(\phi)$ is identically zero.

By identifying $\phi^{*}$ and the scaling behavior in the active jamming regime, the $\ell_{0}$ dependence of the three regions of the swim pressure (dilute, semidilute, and active jamming) can be described quantitatively. From this information, it is straightforward to construct a functional form [70] that captures the full $\phi$ and $\ell_{0}$ dependencies of the swim pressure [69]. In Fig. 2(d), representative curves for the $\phi^{*}$-informed swim pressure are provided for a range of $\ell_{0}$ above the critical intrinsic run length for MIPS. For comparison, we include expressions for the swim pressure put forward in previous works [42,47]. Importantly and in contrast to Refs. [42,47], the swim pressure derived using $\phi^{*}$ does not asymptote to a characteristic functional form at large $\ell_{0}$. In fact, the dependence on $\ell_{0}$ is quite striking but not unexpected given our identified concentration regimes.

The concentration regimes defined above not only describe the swim pressure, but also reveal the $\ell_{0}$ and $\phi$ dependencies of the collisional pressure $\Pi^{c}$, as shown in Figs. 2(e)-2(h) (see Supplemental Material [69] for further discussion). While existing works $[42,47]$ have proposed that $\Pi^{c}$ is independent of $\ell_{0}$, a clear dependence can be observed in Fig. 2(f) and collapsed using $\phi^{*}$ [see Fig. 2(g)]. Deviations from the collapse again occur in the active jamming region, where $\Pi^{c}$ is entirely independent of $\ell_{0}$ and is well described by an EOS for passive polydisperse disks [71] (with $\zeta U_{0}$ replacing the thermal force scale $\left.k_{B} T / \sigma\right)$.

In Fig. 3(a), we compare the total pressure predicted by our dynamic overlap equation of state $\left(\phi^{*}\right.$-EOS) with those derived in previous work. We consider two values of $\ell_{0}$, one near the critical intrinsic run length $\ell_{0} / \sigma=12$ and one deep within the MIPS coexistence region $\ell_{0} / \sigma=40$. At $\ell_{0} / \sigma=12$, the three expressions are found to be in relatively good agreement, while for $\ell_{0} / \sigma=40$ there is a substantial
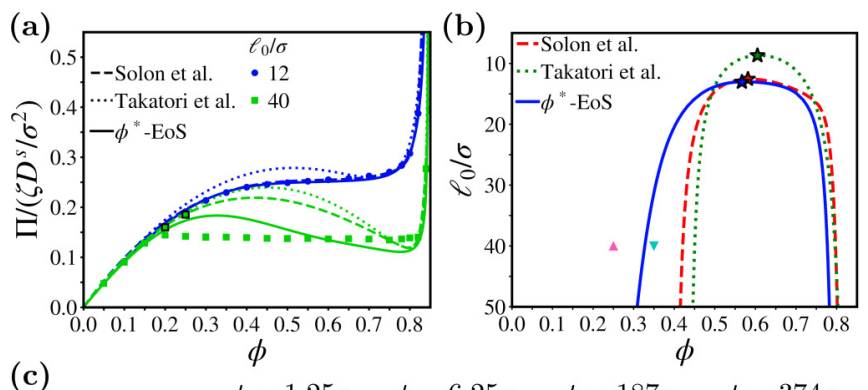

(c)

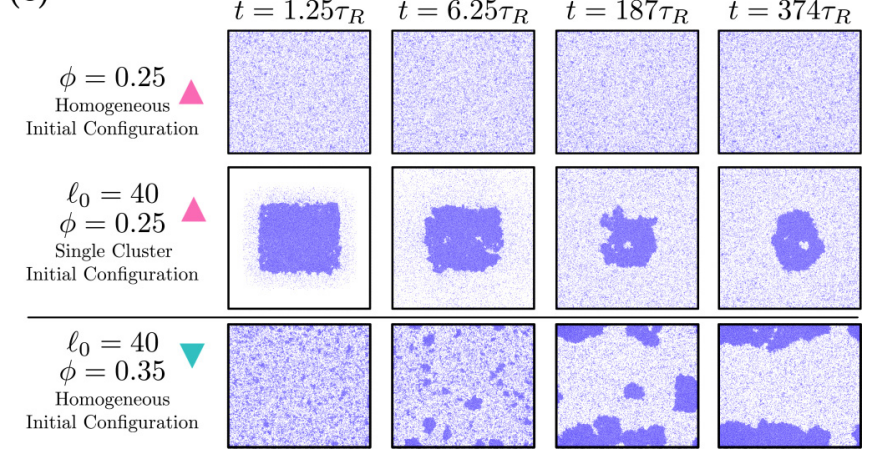

FIG. 3. (a) $\phi^{*}$-EOS in both regions of stability and instability (metastable points are outlined in black) and (b) the predicted spinodal. The $\phi^{*}$-EoS and resulting spinodal are compared to those of Takatori et al. [42] and Solon et al. [47]. Phase separation kinetics (c) confirm that our predicted spinodal indeed delineates regions of the phase diagram in which large wavelength density homogeneity is unstable from regions in which homogeneity is metastable.

disagreement between the $\phi^{*}$-EOS and those proposed in previous works, most notably in the range of concentrations where the equation of state becomes unphysical (manifested as a van der Waals loop) and exhibits a mechanical instability [i.e., $(\partial \Pi / \partial \phi)_{\ell_{0}}<0$ ]. The bounds (the stability limit) of this mechanically unstable region denote the spinodal, shown in full in Fig. 3(b).

Compared to previous works, the $\phi^{*}$-EOS predicts a much broader unstable region- - a prediction that can be directly tested via simulation. In Fig. 3(c), we show a series of snapshots that illustrate the phase separation kinetics for different points in the stability diagram. For the $\left(\ell_{0} / \sigma, \phi\right)$-state-space point $(40,0.35)$, all simulations, independent of the initial configuration, rapidly phase separate [see bottom row of Fig. 3(c)], an indication that this state-space point is unstable (within the spinodal), while, for the $\left(\ell_{0} / \sigma, \phi\right)$-state-space point $(40,0.25)$, it is possible to stabilize both a homogeneous and phase-separated configuration depending on the choice of initial configuration [see the top two rows of Fig. 3(c)]. The metastability at this state-space point demonstrates that it is outside of the spinodal but still within the binodal for MIPS. That these two state points of contrasting stability straddle our predicted spinodal provides an independent verification of the accuracy of $\phi^{*}$-EOS well beyond the critical point. We close by emphasizing that the unique features of the EOS identified in this work were revealed through the recognition of the existence of a dynamic overlap concentration scale. It is our hope and expectation that the use of trajectory-space length scales to define concentration scales will prove to be of utility in the study of nonequilibrium systems. 
S.A.M. acknowledges financial support from the Arnold and Mabel Beckman Foundation. A.K.O. acknowledges support from the Schmidt Science Fellowship in partnership with the Rhodes Trust. J.F.B. acknowledges support by the
National Science Foundation under Grant No. CBET1803662. We gratefully acknowledge the support of the NVIDIA Corporation for the donation of the Titan V GPU used to carry out this work.
[1] Y. Fily, S. Henkes, and M. C. Marchetti, Soft Matter 10, 2132 (2014).

[2] S. A. Mallory, A. Šarić, C. Valeriani, and A. Cacciuto, Phys. Rev. E 89, 052303 (2014).

[3] S. C. Takatori, W. Yan, and J. F. Brady, Phys. Rev. Lett. 113, 028103 (2014).

[4] A. P. Solon, J. Stenhammar, R. Wittkowski, M. Kardar, Y. Kafri, M. E. Cates, and J. Tailleur, Phys. Rev. Lett. 114, 198301 (2015).

[5] A. P. Solon, Y. Fily, A. Baskaran, M. E. Cates, Y. Kafri, M. Kardar, and J. Tailleur, Nat. Phys. 11, 673 (2015).

[6] F. Smallenburg and H. Löwen, Phys. Rev. E 92, 032304 (2015).

[7] R. G. Winkler, A. Wysocki, and G. Gompper, Soft Matter 11, 6680 (2015).

[8] T. Speck and R. L. Jack, Phys. Rev. E 93, 062605 (2016).

[9] M. Joyeux and E. Bertin, Phys. Rev. E 93, 032605 (2016).

[10] Y. Fily, Y. Kafri, A. P. Solon, J. Tailleur, and A. Turner, J. Phys. A: Math. Theor. 51, 044003 (2018).

[11] U. M. B. Marconi, C. Maggi, and M. Paoluzzi, J. Chem. Phys. 147, 024903 (2017)

[12] A. Patch, D. Yllanes, and M. C. Marchetti, Phys. Rev. E 95, 012601 (2017).

[13] S. Das, G. Gompper, and R. G. Winkler, Sci. Rep. 9, 6608 (2019).

[14] J. M. Epstein, K. Klymko, and K. K. Mandadapu, J. Chem. Phys. 150, 164111 (2019).

[15] A. K. Omar, Z.-G. Wang, and J. F. Brady, Phys. Rev. E 101, 012604 (2020).

[16] A. Sokolov, L. D. Rubio, J. F. Brady, and I. S. Aranson, Nat. Commun. 9, 1322 (2018).

[17] Y. Li and P. R. ten Wolde, Phys. Rev. Lett. 123, 148003 (2019).

[18] M. Paoluzzi, R. Di Leonardo, M. C. Marchetti, and L. Angelani, Sci. Rep. 6, 34146 (2016).

[19] C. Wang, Y. K. Guo, W. D. Tian, and K. Chen, J. Chem. Phys. 150, 044907 (2019).

[20] S. C. Takatori and A. Sahu, Phys. Rev. Lett. 124, 158102 (2020).

[21] J. Harder, S. A. Mallory, C. Tung, C. Valeriani, and A. Cacciuto, J. Chem. Phys. 141, 194901 (2014).

[22] M. Zaeifi Yamchi and A. Naji, J. Chem. Phys. 147, 194901 (2017).

[23] R. Ni, M. A. Cohen Stuart, and P. G. Bolhuis, Phys. Rev. Lett. 114, 018302 (2015).

[24] S. A. Mallory, M. L. Bowers, and A. Cacciuto, J. Chem. Phys. 153, 084901 (2020).

[25] M. E. Szakasits, W. Zhang, and M. J. Solomon, Phys. Rev. Lett. 119, 058001 (2017)

[26] A. K. Omar, Y. Wu, Z. G. Wang, and J. F. Brady, ACS Nano 13, 560 (2019).

[27] S. A. Mallory, C. Valeriani, and A. Cacciuto, Phys. Rev. E 92, 012314 (2015).

[28] A. Kaiser and H. Löwen, J. Chem. Phys. 141, (2014).

[29] A. Kaiser, S. Babel, B. ten Hagen, C. von Ferber, and H. Löwen, J. Chem. Phys. 142, 124905 (2015).
[30] Y. Q. Xia, W. D. Tian, K. Chen, and Y. Q. Ma, Phys. Chem. Chem. Phys. 21, 4487 (2019).

[31] S. C. Takatori and J. F. Brady, Curr. Opin. Colloid Interface Sci. 21, 24 (2016).

[32] C. F. Lee, Soft Matter 13, 376 (2017).

[33] M. Rein and T. Speck, Eur. Phys. J. E 39, 84 (2016).

[34] R. Zakine, Y. Zhao, M. Knežević, A. Daerr, Y. Kafri, J. Tailleur, and F. van Wijland, Phys. Rev. Lett. 124, 248003 (2020).

[35] U. M. B. Marconi, C. Maggi, and S. Melchionna, Soft Matter 12, 5727 (2016).

[36] T. Speck, Europhys. Lett. 114, 30006 (2016).

[37] U. M. B. Marconi and C. Maggi, Soft Matter 11, 8768 (2015).

[38] R. Wittmann, F. Smallenburg, and J. M. Brader, J. Chem. Phys. 150, 174908 (2019).

[39] U. M. B. Marconi, A. Puglisi, and C. Maggi, Sci. Rep. 7, 46496 (2017).

[40] J. Rodenburg, M. Dijkstra, and R. van Roij, Soft Matter 13, 8957 (2017).

[41] S. Chakraborti, S. Mishra, and P. Pradhan, Phys. Rev. E 93, 052606 (2016).

[42] S. C. Takatori and J. F. Brady, Phys. Rev. E 91, 032117 (2015).

[43] S. Paliwal, J. Rodenburg, R. van Roij, and M. Dijkstra, New J. Phys. 20, 015003 (2018).

[44] R. Wittkowski, A. Tiribocchi, J. Stenhammar, R. J. Allen, D. Marenduzzo, and M. E. Cates, Nat. Commun. 5, 4351 (2014).

[45] S. C. Takatori and J. F. Brady, Soft Matter 11, 7920 (2015).

[46] B. Partridge and C. F. Lee, Phys. Rev. Lett. 123, 068002 (2019).

[47] A. P. Solon, J. Stenhammar, M. E. Cates, Y. Kafri, and J. Tailleur, New J. Phys. 20, 075001 (2018).

[48] S. Hermann, P. Krinninger, D. de las Heras, and M. Schmidt, Phys. Rev. E 100, 052604 (2019).

[49] D. Levis, J. Codina, and I. Pagonabarraga, Soft Matter 13, 8113 (2017).

[50] S. Hermann, D. de las Heras, and M. Schmidt, Phys. Rev. Lett. 123, 268002 (2019).

[51] E. Tjhung, C. Nardini, and M. E. Cates, Phys. Rev. X 8, 031080 (2018).

[52] Y. Fily and M. C. Marchetti, Phys. Rev. Lett. 108, 235702 (2012).

[53] G. S. Redner, M. F. Hagan, and A. Baskaran, Phys. Rev. Lett. 110, 055701 (2013).

[54] I. Buttinoni, J. Bialké, F. Kümmel, H. Löwen, C. Bechinger, and T. Speck, Phys. Rev. Lett. 110, 238301 (2013).

[55] M. E. Cates and J. Tailleur, Annu. Rev. Condens. Matter Phys. 6, 219 (2015).

[56] A. K. Omar, K. Klymko, T. GrandPre, and P. L. Geissler, Phys. Rev. Lett. 126, 188002 (2021).

[57] P.-G. de Gennes, Scaling Concepts in Polymer Physics (Cornell University Press, Ithaca, NY, 1979).

[58] M. Rubinstein and R. Colby, Polymer Physics (OUP, Oxford, UK, 2003).

[59] Z.-G. Wang, Macromolecules 50, 9073 (2017). 
[60] P. Digregorio, D. Levis, A. Suma, L. F. Cugliandolo, G. Gonnella, and I. Pagonabarraga, Phys. Rev. Lett. 121, 098003 (2018).

[61] J. U. Klamser, S. C. Kapfer, and W. Krauth, Nat. Commun. 9, 5045 (2018).

[62] S. Paliwal and M. Dijkstra, Phys. Rev. Research 2, 012013(R) (2020).

[63] J. D. Weeks, D. Chandler, and H. C. Andersen, J. Chem. Phys. 54, 5237 (1971).

[64] J. A. Anderson, J. Glaser, and S. C. Glotzer, Comput. Mater. Sci. 173, 109363 (2020).

[65] J. Bickmann and R. Wittkowski, Phys. Rev. Research 2, 033241 (2020).

[66] J. Bickmann and R. Wittkowski, J. Phys.: Condens. Matter 32, 214001 (2020).

[67] J. Jeggle, J. Stenhammar, and R. Wittkowski, J. Chem. Phys. 152, 194903 (2020).

[68] T. M. Squires and J. F. Brady, Phys. Fluids 17, 073101 (2005).
[69] See Supplemental Material at http://link.aps.org/supplemental/ 10.1103/PhysRevE.104.044612 for supporting data for the scaling analysis presented in the main text and the precise functional forms of the equations of state, which includes Refs. [42,47,71].

[70] The swim pressure constructed using our approach is well described by the following functional form,

$$
\frac{\Pi^{s}}{\Pi_{0}^{s}}=\frac{\ell}{\ell_{0}}=\left(1-\phi / \phi_{m}\right)\left(1+\sum_{i=1}^{5} a_{i} \phi^{i}\right),
$$

while the collisional pressure is captured with

$$
\Pi^{c}=\frac{b_{2} \phi^{2}+b_{3} \phi^{3}+b_{4} \phi^{4}}{\left(1-\phi / \phi_{m}\right)^{\frac{1}{2}}},
$$

where the coefficients $a_{i}$ and $b_{i}$ are provided in the Supplemental Material [69].

[71] A. Santos, S. B. Yuste, M. López de Haro, and V. Ogarko, Phys. Rev. E 96, 062603 (2017). 